\title{
Pattern of localisation error in patients with stroke to sound processed by a binaural sound space processor
}

\author{
S Sonoda, M Mori, A Goishi
}

\begin{abstract}
Objective-The ability of 46 patients with supratentorial stroke and 15 healthy subjects to localise sounds was tested using an apparatus with headphone and sound space processor.

Methods-With a binaural sound space processor, sounds were randomly presented from seven directions in the $\mathbf{1 8 0}$ degree frontal area of the subject at intervals of 30 degrees. The subject was asked to imagine a clock face through the horizontal plane passing through the subject's ears with 12 o'clock denoting a sound from directly in front of the subject. After each sound, the subject indicated the direction from which he or she thought the sound came by mentioning the corresponding hour hand on the clock face; therefore, the answer directions were also separated by 30 degrees. A total of 21 sounds with three sounds from each direction, were presented in random order. The error between the presented direction and the answered direction of each sound was calculated.
\end{abstract}

Results-The mean absolute error which does not distinguish whether an error was in the counterclockwise or clockwise direction, was larger in the patients with stroke than in the healthy subjects. Overall, the patients with stroke who had right brain damage $(n=29)$ had a larger mean absolute error than those who had left brain damage $(n=17)$. The patients with right brain damage did not show any systematic deviation such as a rightward error or leftward error.

Conclusion-A right brain lesion or left brain lesion can cause a patient to have error in sound localisation, and patients with right brain damage generally have a larger mean absolute error of sound localisation. The difference in the mean absolute error of sound localisation between patients with stroke with right brain damage and those with stroke with left brain damage may be explained by the inattention theory of hemispatial neglect. (f Neurol Neurosurg Psychiatry 2001;70:43-49)

Keywords: sound localisation; cerebrovascular disorders; agnosia

Many aspects of the cognitive function of patients with stroke have not been elucidated. Spatial cognition is a major higher cortical function. Although visual hemispatial neglect in patients with stroke has been well studied, ${ }^{1-3}$ there is little information on the auditory space of patients with stroke. ${ }^{4-9}$

Wortis and Pfeffer presented sounds to a patient with a right brain lesion, and reported that the patient had disorientation in locating sounds from his left side. ${ }^{4}$ Sanchez-Longo and Foster reported that an error in sound localisation existed in 50 patients with stroke. ${ }^{5}$ Klingon and Bontecou showed a close relation between a mistake in sound localisation and right sided brain damage. ${ }^{6}$ It has been shown that patients with stroke show inaccuracy in the sound localisation task.

Some studies have focused on the types of sound localisation errors made by patients with stroke. Ruff et al distinguished the concepts of absolute error and systematic (directional or relative) error of sound localisation. ${ }^{7}$ The absolute error does not take into account the direction of the error, whereas the systematic error shows the tendency of deviation of the answers of a subject towards one direction. Patients with right brain lesions were shown to have a systematic rightward error of sound localisation by Bisiach et $a l,{ }^{8}$ who used headphones, and by Pinek et al, ${ }^{9}$ who used speakers in a free field. On the other hand, Ruff et al could not detect a systematic error in patients with stroke even after stratification of the patients by the location of the lesion. ${ }^{7}$

To solve the discrepancy in previous studies, we analyzed the errors of sound localisation in patients with stroke using the RSS-10 sound space processor and headphones. To study the overall tendency of sound localisation of each person's responses, a cross table between the presented directions and the answered directions was made. In addition, the mean absolute error and mean systematic error were calculated for each patient.

\section{Methods}

SUBJECTS

Forty six patients with supratentorial stroke who did not have hearing difficulty and 15 healthy subjects (mean age, 58 (SD 5) years; five men and 10 women) were included in this study. Twenty five of the 46 patients with stroke had cerebral infarction and 21 had cerebral haemorrhage. Of the 46 patients with stroke, 17 had a left brain lesion (L group; mean age, 57(SD 10) years; 13 men and four women) and 29 patients had a right brain lesion ( $\mathrm{R}$ group; mean age, 60 (SD 12) years; 21 men and eight women). Each patient had only one lesion. No 


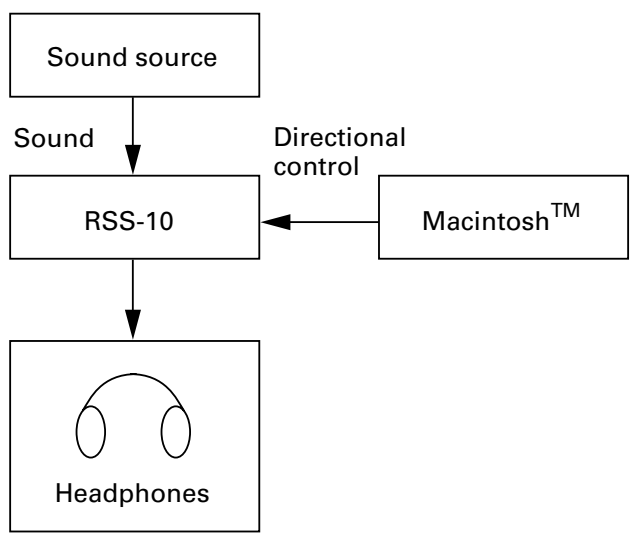

Figure 1 Diagram of the apparatus that produces sounds with directional character in real time.

patient had a binaural difference of hearing difficulty of more than $20 \mathrm{~dB}$ at both $1000 \mathrm{~Hz}$ and $4000 \mathrm{~Hz}$. All of the subjects were alert enough to understand how to execute the hearing test. All could imagine a clockface and draw it on a piece of paper. One patient who could not determine the direction of a sound one time in 27 trials had been excluded in advance. All of the patients with stroke and healthy subjects in the present study could promptly answer with a direction after each sound, irrespective of whether the answer was correct or not.

This study was approved by the human subjects committee at Keio University Tsukigase Rehabilitation Centre. Informed consent was obtained from each subject.

SOUND GENERATION

The RSS-10 sound space processor (Roland Co, Tokyo, Japan) was used to produce sounds that had directional character in real time. The sensation of direction is realised by simulation of the sound transfer function, as well as control of the interaural intensity difference and the interaural time difference. ${ }^{10}$ The program for personal computer (Macintosh) which came with the RSS-10, localises the presented sound. White noise and female voice were used as the sound sources. White noise was continuously generated from the audiogram. The sentence presented by the female voice was, "Hi, I am here. Can you say where I am?" in Japanese. The subject wore a headphone and heard the sound already modified by the RSS-10 (fig 1).

\section{SOUND PRESENTATION}

Sounds were presented through the headphone from directly in front of the subject (midline) and from 30,60, and 90 degrees towards the right and 30,60, and 90 degrees towards the left of the midline (fig 2), three times from each direction for a total of 21 sounds in random order. Twenty one trials of white noise were followed by 21 trials of the female voice. The subject was instructed to localise the sound by imagining a clock face through the horizontal plane passing through the subject's ears (fig 2) and saying the hour number of the clock face from which he or she thought the sound came.

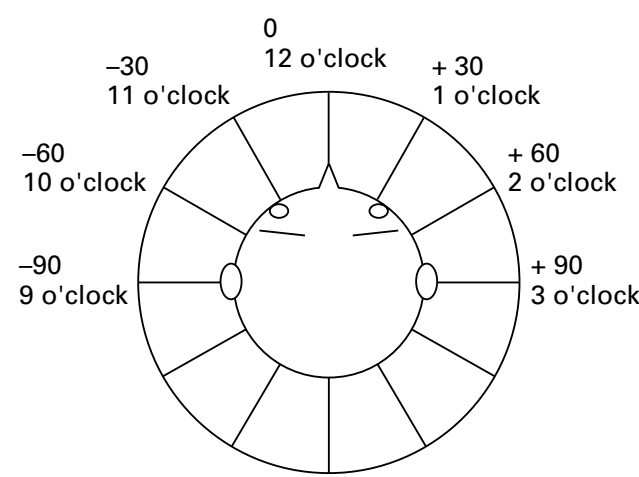

Figure 2 Diagram of directions from which sounds were presented in relation to the subject's head. Sounds were presented from seven directions in the horizontal plane through the subject's ears in the frontal area of the subject at intervals of 30 degrees.

If the subject thought the sound came from directly in front, he or she would say " 12 o'clock". If the subject thought the sound came from the direction of the right ear, the subject would say " 3 o'clock". Therefore, the separation between the presented sounds and the separation between the answered sounds were each 30 degrees.

\section{STATISTICAL ANALYSES}

All statistical analyses were applied to the three groups - that is, the healthy group, L group, and R group, for each of the two sound sources, white noise, and female voice. The frequency of the correct answer to each presented direction was calculated. The frequency of an answered direction that was symmetrically posterior to the presented direction (for example, if the presented sound was 2 o'clock and the subject answered 4 o'clock) was also determined. The frequency of anterior-posterior confusion that sometimes occurs in tests conducted with headphones, was assessed.

In the following analyses, the answered directions that were located in the posterior field were converted to the corresponding anterior direction. That is, 4 o'clock in the raw data was converted to 2 o'clock; 7 o'clock was converted to 11 o'clock etc.

For each patient, we calculated a mean absolute error and mean systematic error using different subsets of the data: we calculated the mean absolute error and mean systematic error using all data on the patient; the mean absolute error and mean systematic error using the data on sounds presented from the left auditory field; and the mean absolute error and mean systematic error using the data on sounds presented from the right auditory field. In this analysis, the left auditory field was defined as sounds presented from 9 o'clock to 11 o'clock; the right auditory field was defined as sounds presented from 1 o'clock to 3 o'clock; and the whole field was defined as sounds presented from all seven directions. The mean absolute error for a subject was defined as the mean of the angle (in degrees) between the presented and answered directions (where each angle $=180$ degrees), irrespective of whether an incorrectly answered position was a counterclockwise error or clockwise error. The mean 
Table 1 Percentage of correct answers in the sound localisation test

\begin{tabular}{|c|c|c|c|c|c|c|c|c|c|c|c|c|c|c|c|c|c|c|}
\hline \multirow[b]{2}{*}{ Group } & \multirow[b]{2}{*}{ Result } & \multirow{2}{*}{$\begin{array}{l}\text { Answered } \\
\text { direction }\end{array}$} & \multicolumn{8}{|c|}{ White noise presented direction } & \multicolumn{8}{|c|}{ Female voice presented direction } \\
\hline & & & -90 & -60 & -30 & 0 & 30 & 60 & 90 & Mean & -90 & -60 & -30 & 0 & 30 & 60 & 90 & Mean \\
\hline \multirow{6}{*}{$\begin{array}{l}\text { Healthy group } \\
\quad(n=15)\end{array}$} & \multirow[t]{3}{*}{ Correct } & Anterior & & 66.7 & 55.6 & 44.4 & 24.4 & 24.4 & & 43.1 & & 48.9 & 42.2 & 15.6 & 8.9 & 26.7 & & 28.4 \\
\hline & & Posterior & & 2.2 & 6.7 & 40.0 & 26.7 & 15.6 & & 18.2 & & 13.3 & 4.4 & 80.0 & 31.1 & 26.7 & & 31.1 \\
\hline & & Total & 51.1 & 68.9 & 62.2 & 84.4 & 51.1 & 40.0 & 71.1 & 61.3 & 73.3 & 62.2 & 46.7 & 95.6 & 40.0 & 53.3 & 66.7 & 62.5 \\
\hline & \multirow[t]{3}{*}{ Incorrect } & Anterior & 42.2 & 13.3 & 20.0 & 4.4 & 20.0 & 6.7 & 13.3 & 17.1 & 20.0 & 17.8 & 31.1 & 0.0 & 11.1 & 2.2 & 22.2 & 14.9 \\
\hline & & Posterior & 6.7 & 0.0 & 15.6 & 11.1 & 24.4 & 2.2 & 15.6 & 10.8 & 6.7 & 0.0 & 22.2 & 4.4 & 44.4 & 0.0 & 11.1 & 12.7 \\
\hline & & Total & 48.9 & 31.1 & 37.8 & 15.6 & 48.9 & 60.0 & 28.9 & 38.7 & 26.7 & 37.8 & 53.3 & 4.4 & 60.0 & 46.7 & 33.3 & 37.5 \\
\hline \multirow[t]{6}{*}{$\mathrm{L}$ group $(\mathrm{n}=17)$} & \multirow[t]{3}{*}{ Correct } & Anterior & & 39.2 & 27.5 & 45.1 & 13.7 & 25.5 & & 30.2 & & 37.3 & 33.3 & 35.3 & 13.7 & 23.5 & & 28.6 \\
\hline & & Posterior & & 17.6 & 11.8 & 17.6 & 13.7 & 17.6 & & 15.7 & & 11.8 & 9.8 & 31.4 & 11.8 & 15.7 & & 16.1 \\
\hline & & Total & 58.8 & 56.9 & 39.2 & 62.7 & 27.5 & 43.1 & 45.1 & 47.6 & 56.9 & 49.0 & 43.1 & 66.7 & 25.5 & 39.2 & 43.1 & 46.2 \\
\hline & \multirow[t]{3}{*}{ Incorrect } & Anterior & 29.4 & 17.6 & 29.4 & 19.6 & 25.5 & 15.7 & 23.5 & 23.0 & 37.3 & 27.5 & 23.5 & 13.7 & 39.2 & 17.6 & 33.3 & 27.5 \\
\hline & & Posterior & 11.8 & 2.0 & 25.5 & 11.8 & 33.3 & 9.8 & 31.4 & 17.9 & 5.9 & 2.0 & 9.8 & 19.6 & 19.6 & 3.9 & 23.5 & 12.0 \\
\hline & & Total & 41.2 & 43.1 & 60.8 & 37.3 & 72.5 & 56.9 & 54.9 & 52.4 & 43.1 & 51.0 & 56.9 & 33.3 & 74.5 & 60.8 & 56.9 & 53.8 \\
\hline \multirow{6}{*}{$R$ group $(n=29)$} & \multirow[t]{3}{*}{ Correct } & Anterior & & 27.6 & 34.5 & 33.3 & 27.6 & 36.8 & & 32.0 & & 31.0 & 25.3 & 25.3 & 8.0 & 17.2 & & 21.4 \\
\hline & & Posterior & & 9.2 & 6.9 & 9.2 & 5.7 & 12.6 & & 8.7 & & 23.0 & 5.7 & 25.3 & 21.8 & 14.9 & & 18.2 \\
\hline & & Total & 29.9 & 36.8 & 41.4 & 42.5 & 33.3 & 49.4 & 47.1 & 40.1 & 37.9 & 54.0 & 31.0 & 50.6 & 29.9 & 32.2 & 42.5 & 39.7 \\
\hline & \multirow[t]{3}{*}{ Incorrect } & Anterior & 46.0 & 29.9 & 39.1 & 36.8 & 31.0 & 17.2 & 32.2 & 33.2 & 39.1 & 19.5 & 33.3 & 19.5 & 31.0 & 11.5 & 31.0 & 26.4 \\
\hline & & Posterior & 24.1 & 3.4 & 11.5 & 13.8 & 17.2 & 4.6 & 20.7 & 13.6 & 23.0 & 6.9 & 23.0 & 20.7 & 24.1 & 12.6 & 25.3 & 19.4 \\
\hline & & Total & 70.1 & 63.2 & 58.6 & 57.5 & 66.7 & 50.6 & 52.9 & 59.9 & 62.1 & 46.0 & 69.0 & 49.4 & 70.1 & 67.8 & 57.5 & 60.3 \\
\hline
\end{tabular}

The percentage of correct and incorrect answers of the subjects in the healthy group, L group or R group, is shown. White noise or female voice was presented to each subject from each of the seven indicated directions three times in random error. An answer in the posterior field that was symmetric around the horizontal line to the presented anterior direction, was considered to be correct.

systematic error for a subject was defined as the mean of the angle (in degrees) between the presented and answered directions in which the direction of the error was considered; the angle (angle $=180$ degrees) of a counter clockwise error was indicated as a negative number, whereas the angle of a clockwise error was indicated as a positive number. An unpaired $t$ test was used to determine significant differences between the healthy group, $\mathrm{R}$ group, and L group.

Cross tables that show the percentage of each particular answer direction to the sounds of each presented direction were made for the three groups. As each subject was presented sounds three times from each of seven directions, 21 data points/subject in the respective group were used to make these tables.

A cross table that showed the percentage of each particular answer direction to the sounds of each presented direction was also made for each subject. From the subject's cross table, the subject's response pattern to each of left auditory presentation and right auditory presentation was classified into one of four patterns: "deviated to the left", "deviated to the right", "bilaterally fluctuated", or "deviated a little". The subject's response to left auditory presentation was classified based on the six answers to the presented sounds at 10 and 11 o'clock, to determine the response tendency to sounds in the left auditory field, and the subject's response to right auditory presentation was classified based on the six answers to the presented sounds at 1 and 2 o'clock, to determine the response tendency to sounds in the right auditory field. "Deviated to the left" or "deviated to the right" was applied to the following four conditions: (1) five or six of the six answers were deviated counter clockwise or deviated clockwise, respectively. (2) Four of the six answers were deviated in the same direction (counter clockwise or clockwise, respectively), and the remaining two answers did not deviate in the opposite direction. (3) At least half of the data (three of six or more) deviated unilaterally 60 degrees or more (counter clockwise or clockwise, respectively), and the error of all other data was 30 degrees or less. (4) Two of the six data deviated greater than or equal to 60 degrees in the same direction (counter clockwise or clockwise, respectively), and none of the remaining data deviated in the opposite direction. "Deviated little" was applied when the error of five or six of the six data was 30 degrees or 0 degrees, and the conditions of "deviated to the left" and "deviated to the right" were not satisfied. The cases that did not meet the criteria of "deviated to the left", "deviated to the right", or "deviated little" were classified as "bilaterally fluctuated".

\section{Results}

Using the RSS- 10 system, 60 of the 61 subjects could feel the sounds extracranially and give an answered direction to all of the presented sounds. When stimulation was applied from 12 o'clock, some L group and R group patients said that the sound came from near the top of the head. The percentage of correct answers in the anterior direction, and the percentage of answers in the posterior direction that were symmetric to the correct anterior direction around the horizontal axis, are shown in table 1 . The rate of correct answers considering posterior answers that were symmetric to the correct anterior answer as correct, to the white noise and female voice was $62 \%$ in the healthy group, $47 \%$ in the L group, and $40 \%$ in the $\mathrm{R}$ group.

The mean values of the mean absolute error and mean systematic error of the subjects in the three groups are shown in table 2. The mean absolute error of the healthy group was significantly smaller than those of the L group and R group to white noise and the female voice, except for left auditory presentation of white noise between the L group and healthy group. The mean absolute error of the $\mathrm{R}$ group was significantly larger than that of the L group only in the conditions of left auditory field presentation of white noise and presentation of white noise from all directions; there were no other significant differences in the mean absolute error of the $\mathrm{R}$ group and $\mathrm{L}$ group. The 
Table 2 Mean absolute error and mean systematic error of sound localisation

\begin{tabular}{|c|c|c|c|}
\hline & \multicolumn{3}{|c|}{ Auditory field presented } \\
\hline & Whole & Left & Right \\
\hline \multicolumn{4}{|l|}{ Mean absolute error: } \\
\hline \multicolumn{4}{|l|}{ White noise } \\
\hline Healthy group & $12.0(3.1)$ & $12.2(6.3)$ & $14.2(5.1)$ \\
\hline L Group & $19.3(6.4)^{\star \star}$ & 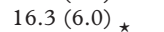 & $22.5(8.1)^{\star \star}$ \\
\hline R Group & $24.2(8.6)^{\star}+\dagger$ & $25.9(10.7)^{\star}+\dagger$ & $21.0(7.3) \mathrm{t \dagger}$ \\
\hline \multicolumn{4}{|l|}{ Female voice } \\
\hline Healthy group & $11.4(4.2)$ & $11.8(6.0)_{\star \star}$ & $14.4(5.1)$ \\
\hline L Group & $21.4(8.8)^{\star \star}$ & $21.4(12.2)^{\star \star}$ & $23.7(8.1)^{\star \star}$ \\
\hline R Group & $25.3(10.8)+\dagger$ & $24.6(12.4)+t$ & $25.6(14.1)+\dagger$ \\
\hline \multicolumn{4}{|c|}{ Mean systematic error: } \\
\hline \multicolumn{4}{|c|}{ White noise } \\
\hline Healthy group & $4.2(4.2)$ & $3.3(8.0)$ & $6.2(6.8)_{\star}$ \\
\hline L Group & $0.3(5.5)^{\star}$ & $1.4(10.5)_{\star}$ & $-1.8(9.1)$ \\
\hline R Group & $5.3(10.1)$ & $12.1(15.5)+$ & $-1.3(13.6)$ \\
\hline \multicolumn{4}{|l|}{ Female voice } \\
\hline Healthy group & $2.7(4.1)$ & $-0.2(6.7)$ & $6.4(7.7)$ \\
\hline L Group & $-0.8(10.2)$ & $3.3(20.0)$ & $-3.3(16.3)^{\star \star}$ \\
\hline R Group & $1.8(10.7)$ & $9.0(16.5)+$ & $-4.0(18.0)+\dagger$ \\
\hline
\end{tabular}

${ }^{\star} \mathrm{p}<0.05 ;{ }^{\star \star} \mathrm{p}<0.01$ between adjacent groups; $\dagger^{\star} \mathrm{p}<0.005 ;+\dagger \mathrm{p}<0.01$ between healthy group and R group; unpaired $t$ test.

Mean systematic error, mean of the angle between the presented and answered positions in which the direction of the error is considered (counter clockwise errors were indicated as negative numbers, and clockwise errors as positive numbers). mean systematic error of the $\mathrm{R}$ group (the answers were deviated to the right) was significantly larger than that of the healthy group to left field presentation of sounds. The mean systematic error of the L group was significantly smaller than that of the healthy group to right field presentation of both the white noise and female voice tests.

Cross tables that show the percentage of each particular answer to the presented sounds from each direction in the healthy group, L group, and $\mathrm{R}$ group, are shown in table 3 . The answers in the posterior direction had been converted to the corresponding anterior direction. Most of the answers of the healthy subjects were correct, which is represented by the diagonal line from the upper left corner to the lower right corner. The answers of the $\mathrm{R}$ group patients were more diffusely distributed.

Table 4 presents the number of subjects in the three groups who showed each particular pattern of deviation of sound localisation to left and right auditory field presentations of white noise and female voice. When the sounds came

Table 3 Pattern of answers

\begin{tabular}{|c|c|c|c|c|c|c|c|c|c|c|c|c|c|c|c|c|c|c|}
\hline & \multicolumn{8}{|c|}{ White noise } & Sum & \multicolumn{8}{|c|}{ Female voice } & \multirow[t]{2}{*}{ Sum } \\
\hline & Pres & & & & & & & & & & & & & & & & & \\
\hline \multirow{8}{*}{$\begin{array}{l}\text { Answered } \\
\text { Healthy group }(n=15)\end{array}$} & & -90 & -60 & -30 & 0 & 30 & 60 & 90 & & & -90 & -60 & -30 & 0 & 30 & 60 & 90 & \\
\hline & -90 & 51 & 18 & 2 & & & & & 71 & -90 & 73 & 20 & & & & & & 93 \\
\hline & -60 & 47 & 69 & 22 & & & & & 138 & -60 & 27 & 62 & 40 & & & & & 129 \\
\hline & -30 & 2 & 13 & 62 & 7 & & & & 84 & -30 & & 18 & 47 & 2 & & & & 67 \\
\hline & 0 & & & 13 & 84 & 2 & & & 100 & 0 & & & 13 & 96 & 4 & & & 113 \\
\hline & 30 & & & & 9 & 51 & 9 & & 69 & 30 & & & & 2 & 40 & 2 & & 44 \\
\hline & 60 & & & & & 42 & 40 & 29 & 111 & 60 & & & & & 51 & 53 & 33 & 138 \\
\hline & 90 & & & & & 4 & 51 & 71 & 127 & 90 & & & & & 4 & 44 & 67 & 116 \\
\hline \multirow[t]{7}{*}{ L Group ( $\mathrm{n}=17)$} & -90 & 59 & 24 & 6 & 4 & & & & 92 & -90 & 57 & 22 & 24 & & 2 & & & 104 \\
\hline & -60 & 33 & 57 & 39 & 4 & & & & 133 & -60 & 29 & 49 & 22 & 12 & & & & 112 \\
\hline & -30 & 8 & 18 & 39 & 6 & & 4 & & 75 & -30 & 6 & 25 & 43 & 10 & 2 & & & 86 \\
\hline & 0 & & & 16 & 63 & 14 & 4 & & 96 & 0 & 6 & 2 & 6 & 67 & 22 & 2 & 2 & 106 \\
\hline & 30 & & 2 & & 12 & 27 & 18 & 16 & 75 & 30 & 2 & 2 & 2 & 8 & 25 & 20 & 18 & 76 \\
\hline & 60 & & & & 10 & 45 & 43 & 39 & 137 & 60 & & & 4 & 4 & 35 & 39 & 37 & 120 \\
\hline & 90 & & & & 2 & 14 & 31 & 45 & 92 & 90 & & & & & 14 & 39 & 43 & 96 \\
\hline \multirow[t]{7}{*}{ R Group (n=29) } & -90 & 30 & 30 & 7 & 1 & 1 & & & 69 & -90 & 38 & 18 & 13 & 6 & & & 1 & 76 \\
\hline & -60 & 38 & 37 & 25 & 14 & 1 & 1 & & 116 & -60 & 33 & 54 & 34 & 11 & 1 & 1 & 1 & 137 \\
\hline & -30 & 28 & 26 & 41 & 9 & 2 & & & 107 & -30 & 21 & 20 & 31 & 8 & 2 & & & 82 \\
\hline & 0 & 2 & 5 & 21 & 43 & 9 & 1 & & 80 & 0 & 7 & 6 & 18 & 51 & 17 & 3 & 6 & 108 \\
\hline & 30 & 1 & 1 & 3 & 16 & 33 & 20 & 10 & 85 & 30 & & & 3 & 11 & 30 & 20 & 13 & 77 \\
\hline & 60 & 1 & 1 & 1 & 11 & 36 & 49 & 43 & 143 & 60 & 1 & 1 & & 9 & 34 & 32 & 37 & 115 \\
\hline & 90 & & & 1 & 6 & 17 & 29 & 47 & 100 & 90 & & 1 & & 3 & 15 & 44 & 43 & 106 \\
\hline
\end{tabular}

Each number in a cross table represents the percentage of a given answer among the answers of the indicated group, to the presented direction. The sum of the numbers in a column of a cross table is 100 .

Table 4 Summary of the patients' response patterns to left auditory and right auditory presentation

\begin{tabular}{|c|c|c|c|c|c|c|c|c|c|c|c|c|c|}
\hline \multicolumn{7}{|l|}{ White noise } & \multicolumn{7}{|l|}{ Female voice } \\
\hline \multirow{7}{*}{$\begin{array}{l}\text { Right auditory } \\
\text { presentation }\end{array}$} & \multicolumn{6}{|c|}{ Left auditory presentation } & $\begin{array}{l}\text { Right auditory } \\
\text { presentation }\end{array}$ & \multicolumn{6}{|c|}{ Left auditory presentation } \\
\hline & & Left & Right & Bilateral & Little & Total & Healthy group & & Left & Right & Bilateral & Little & Total \\
\hline & Left & 0 & 0 & 0 & 0 & 0 & & Left & 0 & 0 & 0 & 0 & 0 \\
\hline & Right & 0 & 0 & 0 & 7 & 7 & & Right & 1 & 0 & 0 & 5 & 6 \\
\hline & Bilateral & 0 & 0 & 0 & 0 & 0 & & Bilateral & 0 & 0 & 0 & 0 & 0 \\
\hline & Little & 1 & 0 & 0 & 7 & 8 & & Little & 1 & 0 & 0 & 8 & 9 \\
\hline & Total & 1 & 0 & 0 & 14 & 15 & & Total & 2 & 0 & 0 & 13 & 15 \\
\hline \multirow[t]{6}{*}{ L Group } & & Left & Right & Bilateral & Little & Total & L Group & & Left & Right & Bilateral & Little & Total \\
\hline & Left & 0 & 0 & 0 & 0 & 0 & & Left & 0 & 0 & 0 & 1 & 1 \\
\hline & Right & 0 & 0 & 0 & 3 & 3 & & Right & 2 & 0 & 0 & 2 & 4 \\
\hline & Bilateral & 0 & 0 & 0 & 3 & 3 & & Bilateral & 0 & 1 & 0 & 0 & 1 \\
\hline & Little & 1 & 0 & 0 & 10 & 11 & & Little & 2 & 0 & 1 & 8 & 11 \\
\hline & Total & 1 & 0 & 0 & 16 & 17 & & Total & 4 & 1 & 1 & 11 & 17 \\
\hline \multirow[t]{6}{*}{ R Group } & & Left & Right & Bilateral & Little & Total & R Group & & Left & Right & Bilateral & Little & Total \\
\hline & Left & 0 & 0 & 0 & 0 & 0 & & Left & 0 & 0 & 0 & 1 & 1 \\
\hline & Right & 1 & 2 & 1 & 2 & 6 & & Right & 2 & 2 & 1 & 3 & 8 \\
\hline & Bilateral & 0 & 0 & 0 & 1 & 1 & & Bilateral & 1 & 0 & 1 & 2 & 4 \\
\hline & Little & 3 & 3 & 2 & 14 & 22 & & Little & 1 & 2 & 1 & 12 & 16 \\
\hline & Total & 4 & 5 & 3 & 17 & 29 & & Total & 4 & 4 & 3 & 18 & 29 \\
\hline
\end{tabular}

In each group, the number of patients with the indicated response patterns to left auditory presentation and to right auditory presentation of the indicated sound, is shown. 
from the right auditory field, in all three groups "deviated right" was seen in more subjects than "deviated left" or "bilaterally deviated", except in the L group to white noise where the same number of patients were "deviated right" or "bilaterally deviated". To left auditory field presentation, most of the subjects in the healthy group and L group had the "deviated little" response; in the $\mathrm{R}$ group, although over $50 \%$ of the patients had a "deviated little" response pattern, about equal numbers of the remaining patients were "deviated left", "deviated right", or "bilaterally deviated".

\section{Discussion}

FEATURES OF THE RSS-10 SYSTEM

We studied the ability of patients with stroke and healthy subjects to localise sound with a new quantitative method using a headphone. The conventional headphone methods for studying the ability of sound localisation have two shortcomings - namely, intracranial localisation and anterior-posterior confusion. Weeks et al recently used a system with the sound transfer function to present the localised sound. ${ }^{11}$ We employed similar equipment, the RSS-10, which simulated the sound transfer function and controlled the interaural intensity/time difference. In systems in which only the interaural intensity/time difference is changed, subjects often feel ringing in the head and do not sense sounds from outside the head. ${ }^{12}$ When a sound is heard not from outside of the head but from in the head, the sound cannot be localised. In the present study, using the RSS-10, no healthy subject complained of difficulty in localising sounds. One patient with stroke who was eliminated from the analysis in advance could not determine the direction of a sound; however, this situation occurred only once in 27 trials in this patient. As the frequency of intracranial localisation was very low, it was concluded that the probability that a subject would feel a sound intracranially with our headphone method is very low.

Even though a sound transfer function was used in our system, anterior-posterior confusion often occurred. Anterior-posterior confusion is the phenomenon in which a sound from the anterior direction is felt from the corresponding posterior direction. If two sound sources are symmetrically located 30 degrees from the right side of the subject toward the anterior and posterior poles, the distances from each sound source to the left and right ears are the same. Therefore, the same interaural time/ intensity difference is obtained from sounds at two source locations. This may cause anteriorposterior confusion. The hemiplegic patients and healthy subjects in the present study showed a similar pattern of anterior-posterior confusion. Considering the cause of the confusion, symmetric conversion of the posterior answer to the corresponding anterior location (posterior-anterior conversion), which we performed in our analysis of the data, is reasonable.

In the present study, the head of the subject was not fixed during the sound localisation testing. In the concept of egocentric space, head centred stimulation and body centred stimulation may have different effects in patients with visual hemispatial neglect. ${ }^{13}$ The method used in the present study can be characterised as head centred because headphones were used. As some patients with left hemispatial neglect syndrome have right neck rotation at rest which cannot be corrected by the patient, the task using speakers cannot be classified into head centred or body centred. Thus, the headphone method is useful in head centred experiments.

VALIDITY OF THE TEST

The sound transfer function of RSS-10 was made by sound sampling using a dummy head. ${ }^{10}$ The validity of our system with RSS-10 in combination with posterior-anterior conversion as a sound localisation test can be verified by comparing the number of accurate answers in the present study with that obtained in previous studies which employed other methods. Inoue et al examined healthy elderly subjects using speakers with sounds that were set at intervals of 30 degrees; $40 \%$ to $50 \%$ of the answers of the subjects were correct. ${ }^{14}$ Our results showed the same or higher rate of correct answers using the same interval of separation of the sound source. Therefore, our system can express directional sensation of the sound to at least a similar degree as that in free field experiments.

SOUND LOCALISATION IN PATIENTS WITH STROKE Some patients with stroke showed a cognitive disturbance of sound localisation that could be detected by our RSS-10 headphone system. What causes this disturbance? The nature of the disturbance can be deduced by distinguishing absolute error and systematic error. The systematic error indicates a consistent counter clockwise or clockwise deviation, and the mean systematic error was calculated in each subject by averaging the data to minimise the random error. By contrast, the magnitude of the absolute error increases with the existence of random error as well as directional error.

We analyzed the pattern of errors in each subject, in addition to calculating the mean absolute error and mean systematic error in each subject, as the calculated mean systematic error includes a "not relative" meaning for the following reason. There is no room for deviation to the left at $\mathbf{- 9 0}$ degrees as a leftward rotation at -90 degrees means the subjective localisation in the posterior field, which is treated in our protocol as answering the corresponding anterior position that is rightward from -90 degrees. Therefore, any misdirection to sounds presented at -90 degrees, even an error of random nature, would result in a rightward deviation. This terminal error effect partly explains the central deviation of the answers of sound localisation in patients with stroke using the speaker method reported by Zatorre et al. ${ }^{15}$ The data on sounds presented at -90 and 90 degrees were excluded in our individual pattern analysis, to minimise the contamination of the mean absolute error into the value of the mean systematic error. 
In the present study, the mean absolute error of the $\mathrm{L}$ group and $\mathrm{R}$ group was each significantly higher than that of the healthy group in nearly all auditory presentations (left, right, whole) of white noise and female voice. It can be concluded that a brain lesion on either side may cause a disturbance in sound localisation. This result is consistent with those of Sanchez-Longo and Foster who studied 50 patients with brain damage using a masking noise. ${ }^{5}$ Because the mean absolute error and mean systematic error of the $\mathrm{R}$ group were significantly larger than those of the L group to left auditory field presentation of white noise, a right brain lesion may cause more severe damage to sound localisation in the contralateral field than a left brain lesion; this is similar to visual hemispatial neglect syndrome. HaeskeDewick et al examined the rate of correct answers of sound direction using speakers placed in a circle, and reported that the patients with right brain damage showed the least accuracy, the patients with left brain damage showed higher accuracy, and the healthy subjects showed the highest accuracy. ${ }^{16}$ This result suggests that the right brain plays a predominant part in sound localisation. Positron emission tomography showed that when discriminating differences in sound direction, the right inferior portion of the posterior parietal cortex is predominantly activated, further supporting our conclusion. ${ }^{10}$

The next step in our analysis was to determine whether the nature of this increased mean absolute error in patients with stroke was systematic or not. Summarising our results on the mean systematic error, left auditory field presentation to the $\mathrm{R}$ group showed a rightward shift, whereas right auditory field presentation to both the R group and L group showed a leftward shift in comparison with the respective value in the healthy subjects. As these rotations are both toward the centre of the field, the terminal error effect mentioned above may have contributed to these results. To determine if these deviations arose from the terminal error effect or not, the pattern of individual deviation, which is less affected by the terminal error effect, was analyzed. Surprisingly, the deviation patterns of the $\mathrm{R}$ group to left auditory field presentation were not unidirectional. In the $\mathrm{R}$ group, the individual deviations were leftward, rightward, and even bilateral (table 4), and this group did not show a particular tendency of deviation. Such heterogeneity of responses to sound localisation was described by Vallar et $a l,{ }^{17}$ who studied the midpoint of the moving sound. The heterogeneity in that study may have arisen from the location of the lesion, or by whether the patient had hemispatial neglect syndrome or not. Further studies with stratification of the patients are needed to elucidate this point. In summary, brain damage increased the random error in the contralateral auditory field, and the terminal error effect was the main factor that contributed to the value of the mean systematic error. Damage to the right brain resulted in more severe error in sound localisation than damage of the left brain.
One possible mechanism of the disturbance of sound localisation in patients with stroke is as follows. Because certain neurons selectively respond to the direction of a sound in a bilateral primary auditory field, ${ }^{18}$ brain damage on either side can cause an increase in the threshold of sound localisation. The difference in the severity of the sound localisation error in patients with damage to the left or right cerebral hemisphere may be explained by one of the hypotheses of visual hemispatial neglect-that is, the inattention theory. ${ }^{1}$ If inattention occurs towards sounds in the left auditory field in the $\mathrm{R}$ group patients, the pattern of error fits our results, in which there was an increase in absolute error without an increase in systematic error. When the concept of hemineglect is applied to our results, we must keep in mind that there are differences between visual conditions and auditory conditions. In visual hemispatial neglect, objects on the left side may disappear in the head. By contrast, a sound from the left is at least heard as a sound even if the direction of the sound is not realised. The difference between disappearing without any effect such as cueing on the one hand, and attracting attention by making a subject listen on the other hand induces a different degree of cueing to the left. In the line bisection task, patients with visual hemispatial neglect checked the midpoint to be further right than the true centre without looking at the left terminal of the line. ${ }^{19}$ However, in the sound localisation task, the test sound itself triggers the attention to the side of the sound. Therefore, if the concept of hemispatial neglect is extended to the auditory aspect, the degree of hemispatial neglect of sound localisation would be less.

Our result in which there was no tendency of subjective rightward deviation of sound cognition in the $\mathrm{R}$ group is contradictory to the result obtained by Bisiach et al, who showed a systematic error in patients with left hemianopsia. ${ }^{8}$ Why were different results obtained? Bisiach et al used the headphone method with interaural intensity difference, and the subject was asked to point to the location on his or her head towards where he or she thought the sound came. ${ }^{8}$ The enhanced reality of our method using simulated sound may have increased the cueing effect to the side from which the sound was presented and obscured the systematic deviation to left field presentation. The longer duration of the sound stimulus in the present study (a continuous sound was presented until the subject gave an answer) than that used in the study of Bisiach et al (2 s) would also contribute to increased information about the sound source. As patients who could not imagine the concept of a clock were not included in the present study, patients with severe hemineglect may have been excluded. Severe hemispatial neglect in a patient may contribute to the systematic error during pointing as a manifestation of visual hemispatial neglect in the study of Bisiach et al, whereas imagining a clockface in our study may be less affected by visual hemispatial neglect. The contamination of visual hemispatial neglect in 
the results of studies on sound localisation in patients with stroke must be considered.

TYPE OF SOUND AND THE ABILITY OF

LOCALISATION

Although the results towards white noise and female voice showed a similar tendency, the difference between the errors of the $\mathrm{R}$ group and $\mathrm{L}$ group was more prominent towards white noise than towards the female voice. White noise covers a wider band of frequencies than the female voice. There are cues for localising sounds in the high frequency area, and other cues for localising sounds in the low frequency area. A wider band sound such as white noise would include much information on sound localisation. In situations where there are many cues, the ability to localise sound is more dependent on how efficiently the subject utilises the cues and is affected to a greater degree by the inattention state. This hypothesis is supported by the finding of a less accurate response of cortical neurons to frequency restricted sound reported by $\mathrm{Xu}$ et al. ${ }^{20}$

A second possibility is that the difference between the errors to white noise and the female voice arose from the contained meaning in the female voice. As meaning is mainly analysed in the left brain, overload of the left brain may worsen the damage to sound localisation in the $\mathrm{L}$ group.

\section{Conclusion}

Some patients with stroke had a large error of sound localisation. A brain lesion on either side may lead to an increase in the mean absolute error of sound localisation, and the error in sound localisation in patients with right brain lesion is probably due to inattention. The error of sound localisation in patients with right brain lesion may represent hemispatial neglect syndrome.
1 Heilman KM, Van Den Abell T. Right hemisphere dominance for attention: the mechanism underlying hemispheric asymmetries of inattention (neglect). Neurology 1980;30:327-30.

2 Kinsbourne M. Mechanisms of unilateral neglect. In: Jeannerod M, ed. Neurophyiological and neuropsychological aspects of spatial neglect. Amsterdam: North-Holland, 1987:69-86.

3 Posner MI, Walker JA, Friedrich FJ, et al. Effects of parietal injury on covert orienting of attention. $f$ Neurosci 1984;4:1863-74.

4 Wortis SB, Pfeffer AZ. Unilateral auditory-spatial agnosia. $\mathcal{F}$ Nerv Ment Dis 1948;108:181-6.

5 Sanchez-Longo LP, Foster FM. Clinical significance of impairment of sound localization. Neurology 1958;8:11925.

6 Klingon GH, Bontecou DC. Localization in auditory space. Neurology 1966;16:879-86.

7 Ruff RM, Hersh NA, Pribram KH. Auditory spatial deficits in the personal and extrapersonal frames of reference due to cortical lesions. Neuropsychologia 1981;19:435-43.

8 Bisiach E, Cornacchia L, Sterzi R, et al. Disorders of perceived auditory lateralization after lesions of the right hemisphere. Brain 1984;107:37-52.

9 Pinek B, Duhamel JR, Cavé C, et al. Audio-spatial deficits in humans: differential effects associated with left versus right hemisphere parietal damage. Cortex 1989;25:175-86.

10 Majima Y, Imai T, Kikumoto T. RSS system: binaural recording method representing three dimensional sound. fapanese Audio Society fournal 1990;30:1-5. (In Japanese.)

11 Weeks RA, Aziz-Sultan A, Bushara KO, et al. A PET study of human auditory spatial processing. Neurosci Lett 1999;262:155-8

12 Yamada K, Kaga K, Uno A, et al. Sound lateralization in patients with lesions including the auditory cortex: comparison of interaural time difference (ITD) discrimination and internaural intensity difference (IID) discrimination. Hearing Res 1996;101:173-80.

13 Farnè $\mathrm{A}$, Ponti $\mathrm{F}$, Làdavas $\mathrm{E}$. In search of biased egocentric reference frames in neglect. Neuropsychologia 1998;36:61123.

14 Inoue J, Okamoto K, Yoshida A, et al. Fundamental research about sound localization; 4th report [abstract]. Audiology fapan 1989;32:673-4. (In Japanese.)

15 Zatorre RJ, Pitto A, Villemure JG. Preserved auditory spatial localization following cerebral hemispherectomy. Brain 1995;118:879-89.

16 Haeske-Dewick H, Canavan AGM, Hömberg V. Sound localization in egocentric space following hemispheric lesions. Neuropsychologia 1996;34:937-42.

17 Vallar G, Guariglia C, Nico D, et al. Spatial hemineglect in back space. Brain 1995;118:467-72.

18 Doan DE, Saunders JC. Sensitivity to simulated directional sound motion in the rat primary auditory cortex. $f$ Neurophysiol 1999;81:2075-87.

19 Ishiai S, Furukawa T, Tsukagoshi H. Visuospatial processes of line bisection and the mechanisms underlying unilateral spatial neglect. Brain 1989;112:1485-502.

$20 \mathrm{Xu} \mathrm{L}$, Furukawa S, Middlebrooks JC. Auditory cortical responses in the cat to sounds that produce spatial illusions. Nature 1999;399:688-91. 